\title{
Contributions of four pain domains to the prediction of patient functioning and pain interference
}

\author{
M. Elena Mendoza, Kevin J. Gertz, and Mark P. Jensen \\ University of Washington, Seattle, WA, USA
}

\begin{abstract}
Pain is a multidimensional experience that can vary in intensity, quality and spatial and temporal characteristics. Although there is a great deal of research supporting the importance of pain intensity as a correlate of patient functioning, there is a lack of research examining the importance of the other components of pain, especially the temporal domain. The purpose of this study was to advance the understanding of the role of four pain domains in predicting both pain interference and psychological functioning in a sample of patients with multiple sclerosis. The findings confirmed the significant association between pain intensity and measures of pain interference and psychological functioning, providing additional support for the importance of assessing pain intensity as a key component of chronic pain assessment. None of the other domains showed statistically significant associations with either of the criterion variables. However, we did find non-significant trends for pain temporal patterns to be associated with depressive symptoms. Specifically, there was a trend for patients reporting constant pain to report more depression than those reporting intermittent and variable pain. This suggests the possibility that the temporal pattern of pain may play a role in the impact of pain on depression; however, more research is needed to confirm this finding. Keywords: pain assessment, pain quality, pain intensity, pain site, pain interference.
\end{abstract}

Received 29 March 2012; received in revised form 31 August 2013; accepted 31 August 2013. Available online 07 March 2014.

\section{Introduction}

Chronic pain is a widespread health problem with major effects on emotional, physical, and cognitive functions and affects everyday social and family life as well as the ability to work (Breivik, Collet, Ventafridda, Cohen, \& Gallacher, 2006). The mechanisms involved in the development of chronic pain are complex and diverse and involve both biological and psychosocial factors. It is associated with specific and non-specific medical conditions such as cancer, osteoarthritis, spinal cord injury, multiple sclerosis, among other medical conditions, and it is a leading cause of disability (Vellucci, 2012).

An adequate assessment of pain using validated instruments is essential for developing successful interventions in pain management so that the interference and negative impact of chronic pain in patients' quality

M. Elena Mendoza, Kevin J. Gertz, and Mark P. Jensen, Department of Rehabilitation Medicine, University of Washington, Seattle, WA, United States. Correspondence regarding this article should be directed to: Mark P. Jensen, Ph.D. Address: Department of Rehabilitation Medicine, Box 359612, University of Washington, Harborview Medical Center, 325 Ninth Avenue, Seattle, WA 98104, USA. Telephone: 206-543-3185. Facsimile: 206-897-4881. E-mail: mjensen@uw.edu of life can be reduced. Nevertheless, the subjective nature of pain as well as its variability can make chronic pain difficult to describe and measure (Gilron \& Jensen, 2011). Moreover, the temporal variability in pain intensity during the day or over longer periods of time also affects the accuracy of the patients' self-reports of their pain (Vellucci, 2012).

Research on pain assessment to date has focused on a number of pain domains, namely, pain intensity, pain quality, pain location, and temporal variations in pain severity. Furthermore, biopsychosocial conceptualizations of chronic pain have examined the effects of factors such as pain related cognitions or attributions, coping strategies, and social support over patients' emotional functioning and the impact of pain in life activity (Hirsh, Kupper, Carter, \& Jensen, 2010). These studies have been carried out in samples of individuals with chronic pain associated with a variety of medical conditions.

Among pain's key domains, pain intensity is the single most widely assessed outcome domain in the clinical chronic pain trials (Litcher-Kelly, Martino, Broderick, \& Stone, 2007; Gilron \& Jensen, 2011). A number of studies have shown that decreases of pain intensity are significantly associated with patients' reports of improvements in their emotional and physical functioning and of decreasing pain interference (Dworkin et al., 2008; van Seventer et al., 2011). 
However, there has been a lack of research addressing the relevance of the other domains of pain. For example, we were able to identify only two recent studies that examined pain quality as it relates to patient functioning. The findings of the first of these demonstrated that items from a pain-quality measure contributed to the prediction of pain interference controlling the effects of pain intensity and unpleasantness (Jensen et al., 2006). A second study demonstrated (for the first time) that changes in measures of pain quality are significantly associated with changes in pain interference and sleep quality over and above the effects of changes in global pain intensity and unpleasantness. These findings support the relevance of assessing pain quality as a component of a complete chronic pain assessment both in research and in clinical practice (Jensen et al., 2010).

A few studies have examined the association between the location of the pain and pain intensity or pain interference (Curtis et al., 1999; Siddall, McClelland, Rutkowski, \& Cousins, 2003; Ullrich, Jensen, Loeser, \& Cardenas, 2008). In addition, more related to the current study, previous research has found that spatial distribution of pain site is associated with psychological distress (Tait, Chibnall, \& Margolis, 1990) and can be considered as a useful clinical diagnostic indicator of psychological disturbance in chronic pain patients (Toomey, Gover, \& Jones, 1983). However, we were unable to identify any research studies that have examined the contribution of the temporal aspect of pain to pain interference and patient functioning.

The purpose of this study was to advance our understanding of the role of four pain domains -namely, pain intensity, pain extent, pain quality, and the temporal aspect of pain -- in predicting both pain interference and psychological functioning in a sample of patients with multiple sclerosis (MS). Based on the results of previous relevant research, we hypothesized the following: (1) greater average pain intensity would be associated with more pain interference and poorer psychological functioning; (2) when controlling for pain intensity, ratings of three pain-quality measures would show significant independent associations with pain interference and psychological functioning; (3) when controlling for pain intensity and pain-quality measures, pain extent would show significant independent associations with the criterion variables. Given the lack of studies examining the role of the temporal aspect of pain in predicting pain interference and/or psychological functioning, we did not have a specific hypothesis concerning how this domain might predict, if it does, the criterion variables. Therefore, we viewed the analyses for examining the latter associations as exploratory.

\section{Methods}

\section{Participants}

Of the 161 participants who were willing to participate in the survey, 40 reported they did not experience any pain other than occasional headaches or menstrual cramps in the past 3 months and therefore were excluded from the sample. As a result, the sample used for analyses consists of 121 participants. Of the 121 participants in our sample, 100 were female (82.6\%). As Table 1 illustrates, the ages of the participants ranged from 19 to 86 years, with a mean age of $54.06(\mathrm{SD}=$ 11.78). The majority of participants were Caucasian $(96.7 \%)$, either married or living with a significant other $(62.0 \%)$, and had at least some post-secondary education $(81.7 \%)$. Thirty-two participants classified themselves as having relapsing remitting MS with symptoms subsiding between attacks, 29 as relapsing remitting MS with symptoms becoming worse following each attack, 30 as secondary progressive MS, 18 as primary progressive MS, and 12 as having progressive-relapsing MS based on a self-reported survey item. All participants provided written consent to participate in the study.

Table 1. Demographic and MS-related information

\begin{tabular}{|c|c|c|}
\hline & Frequency (\%) & $\mathrm{M}(\mathrm{SD})$ \\
\hline Age & Range: $19-86$ & $\begin{array}{l}54.06 \\
(11.78)\end{array}$ \\
\hline Sex (female participants) & $100(82.6)$ & - \\
\hline \multicolumn{3}{|l|}{ Educational level } \\
\hline Grades $10-11$ & $1(.8)$ & \\
\hline $\begin{array}{l}\text { High School graduate or GED } \\
\text { recipient }\end{array}$ & $11(9.1)$ & - \\
\hline Vocational or technical school & $10(8.3)$ & - \\
\hline Some college & $28(23.1)$ & \\
\hline College graduate & $47(38.8)$ & - \\
\hline Graduate or Professional School & $24(19.8)$ & \\
\hline \multicolumn{3}{|l|}{ Marital status } \\
\hline Never married & $9(7.4)$ & - \\
\hline Married/ living with significant other & $75(62.0)$ & - \\
\hline Separated/Divorced & $28(23.1)$ & - \\
\hline Widowed & $9(7.4)$ & - \\
\hline \multicolumn{3}{|l|}{ Racial/ethnic group ${ }^{*}$} \\
\hline African-American & $1(0.8)$ & - \\
\hline Asian & $2(1.6)$ & - \\
\hline Caucasian (white) & $117(96.7)$ & - \\
\hline $\begin{array}{l}\text { Native American/American Indian/ } \\
\text { Alaskan Native }\end{array}$ & $3(2.5)$ & - \\
\hline Pacific Islander & $1(0.8)$ & \\
\hline \multicolumn{3}{|l|}{ Course of MS } \\
\hline $\begin{array}{l}\text { Relapsing remitting (symptoms } \\
\text { subside between attacks) }\end{array}$ & $32(26.4)$ & - \\
\hline $\begin{array}{l}\text { Relapsing remitting (worse follow- } \\
\text { ing attacks) }\end{array}$ & $29(24.0)$ & - \\
\hline Secondary progressive & $30(24.8)$ & - \\
\hline Primary progressive & $18(14.9)$ & - \\
\hline Progressive-relapsing & $12(9.9)$ & \\
\hline
\end{tabular}

*Total exceeds 121 because participants were given the option of selecting more than one racial/ethnic group.

\section{Procedures}

Approval for the study was obtained from the University of Washington Institutional Review Board. A pencil-and-paper survey that included the measures described below was sent to 381 individuals with MS. The survey packet included the survey, an invitation letter and consent materials. The individuals approached 
fulfilled at least one of the following: (1) completed a previous survey and indicated they were interested in learning about further research opportunities; (2) learned about the study from another research study conducted by faculty within the Department of Rehabilitation Medicine at the University of Washington (UW); (3) been a patient at the UW Multiple Sclerosis clinic; or (4) contacted research staff upon seeing a recruitment description of the study (i.e., self-referral). Inclusion criteria for the study consisted of the following: (1) being at least 18 years of age; (2) diagnosis of MS from a medical provider; (3) ability to read and write English; and (4) reporting experiencing pain (in addition to occasional headaches or menstrual cramps) in the past 3 months.

\section{Measures}

\section{Demographic information}

Participants were asked to provide basic demographic information including age, gender, educational level and marital status.

\section{MS-related information}

Participants were asked to select a diagram and description that best described their course of MS: relapsing remitting MS with symptoms subsiding between attacks, relapsing remitting MS with symptoms becoming worse following each attack, secondary progressive MS, primary progressive MS, and progressive-relapsing MS.

\section{Pain intensity}

Participants were asked to select a single number on an 11-point numerical rating scale (NRS) including integers from 0 (representing "No pain"), through 10 (representing "Pain as bad as could be") to indicate their average pain intensity in the past week. The $0-10$ NRS has consistently shown its validity as a measure of pain intensity given its strong association with other measures of pain intensity (Jensen \& Karoly, 2011).

\section{Pain interference}

Pain interference was assessed with a modified version of the Pain Interference Scale of the Brief Pain Inventory (BPI-IS, Cleeland \& Ryan, 1994). The original BPI-IS assesses pain interference across seven daily activities (general activity, mood, walking ability, normal work, relations with other people, sleep, and enjoyment of life) on an 11-point numerical rating scale consisting of integers from 0 (representing "does not interfere") through 10 (representing "completely interferes"). In our previous research with individuals with disabilities and pain, we added an additional five items to assess perceived pain interference for the following additional functional domains: self-care, recreational activities, social activities, communication with others, and learning new information and skills (Osborne, Raichle, Jensen, Ehde \& Kraft, 2006; Raichle,
Osborne, Jensen \& Cardenas, 2006). In addition, the walking ability variable was revised to read "Mobility (ability to get around)" to be more accommodating of individuals with physical limitations. The final BPIIS mean score ranges from 0 to 10 , with higher scores indicating greater pain interference. The original scale has shown excellent psychometric properties in the general population (Cleeland \& Ryan, 1994; Jensen \& Karoly, 2011). In addition, the 12-item version has also been shown to be reliable and valid in both spinal cord injury (SCI) and MS populations (Osborne et al., 2006; Raichle et al., 2006).

\section{Descriptive pain measures}

Participants provided information about the extent of their pain, i.e., the presence of persistent, bothersome pain in different locations of the body. A checklist of 18 possible body sites including "other" was used. A measure of pain extent was then computed as the sum of the number of body areas in pain (possible range $=0$ to 18 ; actual range $=0$ to 15 ). In addition, participants were asked about the temporal pattern of their pain with three possible response choices: intermittent (pain comes and goes with some pain-free periods), variable (pain always present but varies in intensity), or constant (pain always present with same intensity). Finally, participants were asked to describe the different qualities or characteristics of their pain using the Pain Quality Assessment Scale (PQAS; Jensen et al., 2006). The PQAS consists of 20 items that describe pain quality domains (e.g., tender, throbbing, heavy). Participants were asked to rate on a 0 to10 NRS how much their pain matched that specific quality domain in the past week, with $0=$ "Not [pain quality]" or "No Pain" to $10=$ "The most [pain quality] sensation imaginable." One study found that, of all the existing pain quality measures, only the PQAS included the 14 pain quality domains most often referenced by participants with MS or spinal cord injury (SCI) who participated in the study (Lin, Kupper, Gammaitoni, Galer \& Jensen, 2011). Researchers have identified three pain quality subgroups or clusters included within the 20 PQAS items: paroxysmal (e.g., shooting, sharp), surface (e.g., itchy, sensitive, cold), and deep (e.g., aching, dull, cramping; Victor et al., 2008). The scale score for each of these clusters is the mean of the items associated with each cluster.

\section{Depression}

The PHQ-9 depression scale was used as a measure of psychological functioning (Kroenke, Spitzer, \& Williams, 2001). It is a 9-item depression module from the full Patient Health Questionnaire (Spitzer, Kroenke, \& Williams, 1999) assessing depression severity. Participants were asked to rate the frequency of nine different symptoms of depression on a 4-point Likert scale rating. The final sum score ranges from 0 to 27 , with higher scores indicating greater severity of depression. The PHQ-9 has been shown to be a reliable and valid measure of depression severity (Kroenke et 
al., 2001). For the current sample, Cronbach's alpha of the PHQ-9 was .64.

\section{Data analyses}

\section{Predicting pain interference}

Hierarchical regression analyses were conducted to examine the extent to which pain intensity, pain quality, the extent of pain, and the temporal aspect of pain predicted pain interference. In these analyses, the BPIIS score was used as the criterion variable. Average pain intensity was entered as the control variable in step 1. Next, the three PQAS scale scores (paroxysmal, surface and deep) were entered as predictor variables in step 2. The extent of pain or total number of body sites participants indicated they experienced pain were entered in Step 3. Finally, three dummy variables representing the different temporal qualities of pain (intermittent, variable, constant) were entered in step 4.

\section{Predicting depression}

Hierarchical regression analyses were also conducted to examine the extent to which pain intensity, pain quality, the extent of pain, and the temporal aspect of pain predicted depression. In these analyses, the PHQ9 mean score was used as the criterion variable. The remaining steps of the model followed the same design as the model constructed to predict pain interference described above.

\section{Results}

\section{Predicting pain interference}

In the hierarchical regression analyses predicting pain interference (Table 2), pain intensity explained $14 \%$ of the variance $(\mathrm{P}<.001)$ in step 1 . The direction of the association suggests that more pain intensity is associated to more pain interference. After controlling for change in pain intensity, none of the other predictors (including pain quality, pain extent, or measures representing the temporal quality of pain) made an additional significant contribution to the prediction of pain interference.

\section{Predicting depression}

Psychological functioning served as the dependent variable in the second hierarchical regression model (Table 3). In step 1, pain intensity accounted for 19\% of the variance in psychological functioning $(\mathrm{P}<.001)$ and the direction of the association suggests that greater average pain intensity during the preceding week is associated with higher scores in severity of depression. None of the other predictors made a significant contribution to the prediction of depression. However, there was a non-significant $(\mathrm{P}<0.10)$ trend for two of the variables representing the temporal components of pain to be associated with depression.

To help interpret this finding, we examined the mean PHQ-9 scores of the participants who selected each of
Table 2. Regression analyses predicting pain interference

\begin{tabular}{|c|c|c|c|c|}
\hline Step and variable & $\mathbf{R}^{2}$ & $\Delta \mathbf{R}^{2}$ & $\Delta \mathrm{F}$ & $\begin{array}{l}\beta \text { to } \\
\text { enter }\end{array}$ \\
\hline Step 1: Pain intensity & .14 & .14 & $18.87^{\mathrm{a}}$ & $.37^{\mathrm{a}}$ \\
\hline Step 2: Pain quality & .16 & .02 & .89 & \\
\hline Paroxysmal pain & & & & -.12 \\
\hline Surface pain & & & & .14 \\
\hline Deep pain & & & & .06 \\
\hline Step 3: Pain extent & .16 & .00 & .45 & .07 \\
\hline Step 4: Temporal quality & .17 & .00 & .22 & \\
\hline Pain that comes and goes & & & & .08 \\
\hline $\begin{array}{l}\text { Constant pain with varia- } \\
\text { tion in intensity }\end{array}$ & & & & .01 \\
\hline $\begin{array}{l}\text { Constant pain with little } \\
\text { variation in intensity }\end{array}$ & & & & .06 \\
\hline
\end{tabular}

Table 3. Regression analyses predicting psychological functioning

\begin{tabular}{|c|c|c|c|c|}
\hline Step and variable & $\mathbf{R}^{2}$ & $\Delta \mathrm{R}^{2}$ & $\Delta \mathbf{F}$ & $\begin{array}{l}\beta \text { to } \\
\text { enter }\end{array}$ \\
\hline Step 1: Pain intensity & .19 & .19 & $27.74^{\mathrm{a}}$ & $.44^{\mathrm{a}}$ \\
\hline Step 2: Pain quality & .21 & .02 & .93 & \\
\hline Paroxysmal pain & & & & -.03 \\
\hline Surface pain & & & & .10 \\
\hline Deep pain & & & & .12 \\
\hline Step 3: Pain extent & .23 & .02 & 2.52 & .15 \\
\hline Step 4: Temporal quality & .25 & .03 & 1.34 & \\
\hline Pain that comes and goes & & & & $-.42^{b}$ \\
\hline $\begin{array}{l}\text { Constant pain with variation } \\
\text { in intensity }\end{array}$ & & & & $-.40^{\mathrm{b}}$ \\
\hline $\begin{array}{l}\text { Constant pain with little } \\
\text { variation in intensity }\end{array}$ & & & & -.21 \\
\hline
\end{tabular}

the four possible temporal patterns. These means were $1.75(\mathrm{SD}, 1.71, \mathrm{~N}=4), 0.88(\mathrm{SD}, 0.94, \mathrm{~N}=66), 1.24$ (SD, 1.18, N = 41), and 1.11 (SD, 0.78, N = 9), for those endorsing no pain in the past 4 weeks, pain that comes and goes (with some pain-free periods), constant pain (with variation in intensity) and constant pain (with little variation), respectively. These means indicate that for participants reporting no pain in the past four weeks, depression severity was mild to moderate, on average. Most of the participants who indicated that their pain comes and goes reported mild depressive symptoms on average, ranging from minimal to moderate depression severity; none reported severe depression. Participants who reported constant pain with variation in intensity also had a mild score in depression severity on average, ranging from minimal to moderate severity. Finally, participants in the group of constant pain with little 
variation reported mild depression severity on average, and none of them had a moderate or severe PHQ-9 score.

\section{Discussion}

The aim of the present study was to examine the relevance of four pain domains in predicting patient psychological functioning and pain interference, in order to know which of those domains, if any, should be routinely assessed in chronic pain clinical practice and research. The findings confirmed the significant association between measures of pain intensity and measures of pain interference and psychological functioning that has been demonstrated in previous research. The direction of the association was consistent with our hypothesis; that is, greater average pain intensity predicts more pain interference and poorer psychological functioning. The results support the importance of assessing pain intensity as a key component of chronic pain assessment.

Although two previous studies found that pain quality measures make significant independent contribution to pain interference in patients with osteoarthritis, low back pain, peripheral neuropathy and carpal tunnel syndrome (Jensen et al., 2006, 2010), this result was not replicated in this study. In addition, and also inconsistent with our hypothesis, the third domain, pain extent, did not show significant associations with any of the criterion variables. Given the paucity of previous research regarding the importance of pain quality and pain extent as predictors of patient functioning, we are unable to determine definitively the reasons that our findings regarding these two pain domains are inconsistent with previous research. One possibility may be that these pain domains are generally important, but that their importance did not emerge in the current study because of chance (an effect that is significant in the population will occasionally emerge as non-significant in a sample due to random variation; Type II error). Alternatively, it is possible that these two domains are generally not important, and that the significant findings by previous researchers emerged due to chance (Type I error). A third possibility-and one that is consistent with our clinical experience-is that the importance of pain quality and pain extent may vary as a function of contextual factors. For example, it is possible that these factors play a larger role in some populations than others. Additional research is needed to help determine which of these explanations is most accurate, and if the third explanation appears correct, to identify the contextual factors (e.g., pain diagnosis or type, demographic factors, etc.) that moderate the associations between these pain domains and patient functioning.

Although pain temporal patterns did not make a significant contribution to the prediction of pain interference or psychological functioning, we identified some non-significant trends that may serve as a reference for future studies. Specifically, the findings indicated that participants with no pain during the past 4 weeks reported more depression than those with constant pain, and that those reporting constant pain scored higher in depressive symptoms than participants with variable pain. Findings regarding the no-pain group are difficult to interpret due to the very small sample size $(\mathrm{N}=4)$ of participants in this category. However, findings related to the constant vs. variable pain groups are intriguing and suggest that the temporal pattern of pain may play some role in the impact of pain on depression. Specifically, having constant pain may lead to more depression than having intermittent and variable pain, perhaps due to the fact that those with more variable pain may have periods of (relative) pain relief. More research is needed with larger samples to explore this possibility further and to determine the role of the temporal patterns in the impact of pain.

This study has several limitations that should be considered. First, the use of self-report measures alone may increase significant association among variables due to shared method variance, which lead to overestimate true relationships. Likewise, use of a singleitem recall measure of pain intensity is a limitation of the study due to research that shows that people tend to over-estimate past pain when using such measures (e.g., Jensen, Mardekian, Lakshminarayanan, \& Boye, 2008). However, even if such measures over-estimate pain in groups of individuals, a great deal of research indicates that they contain valid variance. For example, they demonstrate strong correlations with measures of past pain as measured by daily diaries. They are sensitive to pain treatment and-importantly for the purposes of this study-they are strong correlates of key pain-related domains (Jensen \& Karoly, 2011). Consistent with this research, our measure of pain intensity was strongly associated with both criterion measures in our study.

Also, the current sample included only patients with MS who were willing to complete a survey; individuals with MS who were not interested in completing the survey might differ in some unknown way from the current sample. This may limit the generalizability of the findings to other patients with MS. Furthermore, MS patients differ from other chronic patients in many ways. Likewise, this sample consisted primarily of Caucasian women; other races and ethnicities as well as males were under-represented. Thus, these results do not necessarily address the importance of pain domains as predictors of patient functioning in other chronic pain populations. Another limitation is that the cross-sectional nature of the analyses precludes direct interpretations of causality, so future studies using longitudinal designs would be needed to help understand causal relationships. Also, although we found a non-significant trend for the pain temporal pattern to be associated with depression (with some indication that pain that is constant may be associated with more depression than pain that is variable), the low sample size precluded our ability to confirm this trend. More research is needed with larger samples to provide a more definitive test of these observations. Finally, 
given the lack of research reporting to have examined the role of the temporal aspect of pain on patients' functioning and pain interference, we did not have a priori predictions concerning this role; another reason to consider these results as preliminary. More research is needed to confirm (or disconfirm) our results.

Despite the study's limitations, the findings provide new information regarding the role that the temporal pattern of pain may play in the impact of pain on depression. Although we did not find statistically significant effects, the non-significant trends suggest that the temporal aspect of pain can be potentially important for some patients; therefore, it may be worth assessing in clinical and research settings.

\section{Acknowledgments}

The first author's participation was funded by the Spanish Foundation of Science and Technology (Spanish Ministry of Science and Innovation). This research was supported by grant no. P01 HD33988 from the National Institutes of Health, National Institute of Child Health and Human Development (National Center for Medical Rehabilitation Research), and grant no. H133B031120 from the National Institute of Disability and Rehabilitation Research, Department of Education.

\section{References}

Breivik, H., Collet, B., Ventafridda, V., Cohen, R., Gallacher, D. (2006). Survey of chronic pain in Europe: prevalence, impact on daily life, and treatment. European Journal of Pain, 10, 287-333.

Cleeland C. S. \& Ryan, K. M. (1994). Pain Assessment: Global Use of the Brief Pain Inventory. Annals of the Academy of Medicine, 23(2), 129-138.

Dworkin, R. H., Turk, D. C., Wyrwich, K. W., Beaton, D., Cleeland, C. S., Farrar, J.T., ... Zavisic, S. (2008). Interpreting the clinical importance of treatment outcomes in chronic pain clinical trials: IMMPACT Recommendations. The Journal of Pain, 9, 105-121.

Curtis, K. A., Drysdale, G. A., Lanza, R. D., Kolber M., Vitolo, R. S.,West, R. (1999). Shoulder pain in wheelchair users with tetraplegia and paraplegia. Archives of Physical Medicine and Rehabilitation, 80, 453-457.

Gilron, I., \& Jensen, M. P. (2011). Clinical trial methodology of pain treatment studies: Selection and measurement of self-report primary outcomes for efficacy. Regional Anesthesia and Pain Medicine, 36, 374-381.

Hirsh, A. T., Kupper, A. E, Carter, G. T., \& Jensen, M. P. (2010). Psychosocial factors and adjustment to pain in individuals with postpolio syndrome. American Journal of Physical Medicine and Rehabilitation, 89, 213-224.

Jensen, M. P., Dworkin, R. H., Gammaitoni, A. R., Olaleye, D. O., Oleka, N., Galer, B. S. (2006). Do pain qualities and spatial characteristics make independent contributions to interference with physical and emotional functioning? The Journal of Pain, 7, 644-653.
Jensen, M. P., Gould, E. M., Victor, T. W., Gammaitoni, A. R., White, R. E., \& Galer, B. S. (2010). The relationship of changes in pain quality to pain interference and sleep quality. Official journal of the American Pain Society, 11, 782-788.

Jensen, M. P., \& Karoly, P. (2011). Self-report scales and procedures for assessing pain in adults. In D. C. Turk \& R. Melzack (Eds.) Handbook of pain assessment. (3rd ed., pp. 19-44). New York: Guilford Press.

Jensen, M. P., Mardekian, J., Lakshminarayanan, M., \& Boye, M. E. (2008). Validity of 24-hour recall ratings of pain severity: Biasing effects of "peak" and "end" pain. Pain, 137, 422-427.

Kroenke K., Spitzer R. L., \& Williams, J. B. (2001). The PHQ-9: Validity of a brief depression severity measure. Journal of General Internal Medicine, 16(9), 606-613.

Lin, C. P., Kupper A. E., Gammaitoni A. R., Galer B. S., \& Jensen M. P. (2011). Frequency of chronic pain descriptors: Implications for assessment of pain quality. European Journal of Pain, 15(6), 628-633.

Litcher-Kelly, L., Martino, S. A., Broderick, J. E., Stone, A. A. (2007). A systematic review of measures used to assess chronic musculoskeletal pain in clinical and randomized controlled clinical trials. The Journal of Pain, 8, 906-913.

Osborne, T. L., Raichle, K. A., Jensen, M. P., Ehde, D. M., \& Kraft, G. (2006). The reliability and validity of pain interference measures in persons with multiple sclerosis. Journal of Pain and Symptom Management, 32(3), 217-229.

Raichle, K. A., Osborne, T. L., Jensen, M. P., \& Cardenas, D. (2006). The reliability and validity of pain interference measures in persons with spinal cord injury. Journal of Pain, 7(3), 179-186.

Siddall, P. J., McClelland, J. M., Rutkowski, S. B., \& Cousins, M. J. (2003). A longitudinal study of the prevalence and characteristics of pain in the first 5 years following spinal cord injury. Pain, 103, 249-257.

Spitzer, R. L., Kroenke K., \& Williams, J. B. (1999). Validation and utility of a self-report version of PRIME-MD: The PHQ primary care study. The Journal of the American Medical Association (JAMA), 282(18), 1737-44.

Tait, R. C., Chibnall, J. T., \& Margolis, R. B. (1990). Pain extent: Relations with psychological state, pain severity, pain history, and disability. Pain, 41, 295-301.

Toomey, T. C., Gover, V. F., \& Jones, B. N. (1983). Spatial distribution of pain: A descriptive characteristic of chronic pain. Pain, 17, 289-300.

Turk, D. C., Dworkin, R. H., Allen, R. R., Bellamy, N., Brandenburg, N., Carr, D. B., ...Witter, J. (2003). Core outcome domains for chronic pain clinical trials: IMMPACT recommendations. Pain, 106, 337-345, 2003

Ullrich, P. M, Jensen, M. P., Loeser, J. D, \& Cardenas, D. D. (2008). Pain intensity, pain interference and characteristics of spinal cord injury. Spinal Cord, 46, 451-455.

Van Seventer, R., Serpell, M., Flemming, W. B., Morlion, B., Zlateva, F. Bushmakin, A.G., ... Nimour, M. (2011). Relationships between changes in pain severity and other patient-reported outcomes: An analysis in patients with posttraumatic peripheral neuropathic pain. Health and Quality of Life Outcomes, 9, 17, 1-8.

Vellucci, R. (2012). Heterogeneity of chronic pain. Clinical Drug Investigation, 32(Suppl. 1), 3-10.

Victor, T. W., Jensen, M. P., Gammaitoni, A. R., Gould, E. M., White, R. E., \& Galer, B. S. (2008). The dimensions of pain quality: Factor analysis of the Pain Quality Assessment Scale. Clinical Journal of Pain, 24 (6), 550-555. 